\title{
AV Nodal Reentrant Tachycardia with a Bystander Mahaim Fiber
}

\author{
Yoshifusa Aizawa, M.D., Kaoru Suzuki, M.D., \\ Masaomi Ciminusir, M.D., and Akira Simbata, M.D.
}

\begin{abstract}
SUMMARY
A 13 year old boy had a wide QRS complex tachycardia. A discontinuity in the AV nodal functional curve was observed in the electrophysiologic study. The AV interval was prolonged in association with progressive ventricular preexcitation. At maximal preexcitation, the HV interval was $-20 \mathrm{msec}$ and the QRS complex was identical to that seen during clinical tachycardia. No VA conduction was found and atrial premature beats did not affect the tachycardia. The His deflection was found at variable timing when tachycardia was induced. These findings confirmed that tachycardia originated within the AV node and was conducted to the ventricle over the Mahaim fiber. The short effective period of the Mahaim fiber had clinical significance since when atrial fibrillation developed, a rapid ventricular response was observed.
\end{abstract}

\section{Additional Indexing Words:}

Nodo-ventricular fiber AV nodal reentrant tachycardia Electrophysiologic study

$I^{\prime}$

N AV nodal reentrant tachycardia, the conduction to the ventricle may take place over the Mahaim fiber resulting in a wide QRS complex tachycardia, but this occurs only rarely. ${ }^{\text {n-5) }}$ In this paper, we describe a case of a wide QRS complex tachycardia of AV nodal origin conducted over the Mahaim fiber. The Mahaim fiber had a short effective refractory period which caused a rapid ventricular response when atrial fibrillation developed.

\section{Case Report}

The patient was a 13 year old boy. The family history was noncontributory. At the age of 7 , he developed syncope. Since that time, he had had attacks of palpitations which lasted for several minutes, about once a year.

From the First Department of Internal Medicine, Niigata University School of Medicine, Niigata, Japan.

Address for reprints: Yoshifusa Aizawa, M.D., First Department of Internal Medicine, Niigata University School of Medicine, Asahimachi-1, Niigata 951, Japan.

Received for publication October 15, 1987.

Accepted May 23, 1988. 
On the last visit to a doctor, the electrocardiogram (ECG) showed a wide QRS complex tachycardia for the first time. The tachycardia exhibited a rate of 230 beats/min and a QRS complex compatible with left bundle branch block (LBBB) and left axis deviation. Procainamide and lidocaine were ineffective but verapamil reduced the rate to 190 beats $/ \mathrm{min}$. DC shock was delivered to restore normal sinus rhythm. He was then referred to our hospital for further evaluation.

The physical examination on admission disclosed no abnormality. Blood pressure was $136 / 70 \mathrm{mmHg}$ and pulse rate was 78 beats/min. ECG showed normal sinus rhythm and the PR interval was $0.16 \mathrm{sec}$. The QRS complex was narrow and normal in morphology. Chest X-ray showed normal heart and normal lungs. Routine hematological and blood chemistry studies were normal. Echocardiogram was also normal.

The electrophysiologic study:

After obtaining informed consent, the study was performed in the nonsedated postabsorptive state as previously described ${ }^{61,7)}$

The heart was stimulated at the right atrium and the AV nodal functional curve and the refractory periods were obtained. Both AH and HV intervals were normal during normal sinus rhythm, 140 and $55 \mathrm{msec}$, respectively. During the determination of the refractory period at the basic cycle length of $400 \mathrm{msec}$, a discontinuity in the AV nodal functional curve was observed; a sudden prolongation of the AH interval from 320 to $360 \mathrm{msec}$ was found when the coupling inteival of the premature stimulus was reduced by $10 \mathrm{msec}$. At the basic cycle length of $500 \mathrm{msec}$, a sudden prolongation

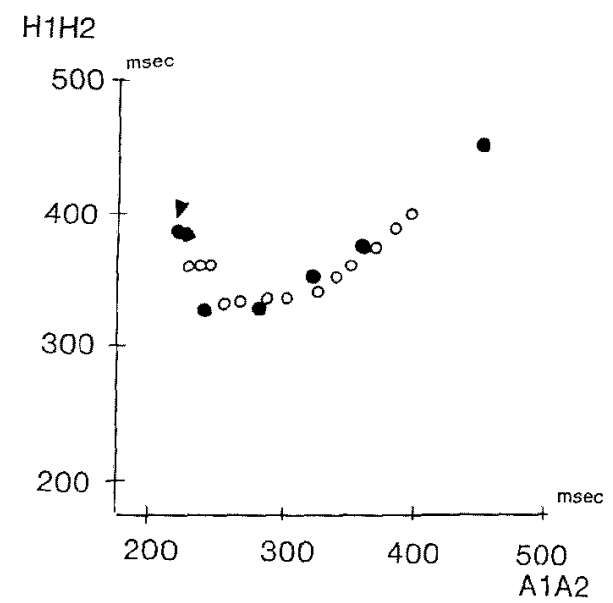

Fig. 1. AV nodal functional curve. A sudden prolongation in the $\mathrm{AH}$ interval was found. $O$ and $O$ : basic cycle lengths at 400 and $500 \mathrm{msec}$, respectively. Tachycardia was induced at arrow. 
of the AH interval by $50 \mathrm{msec}$ was observed with the shortening of the coupling interval of the premature stimulus by $10 \mathrm{msec}$ (Fig. 1).

As the interval of the premature stimulus (AIA2) became shorter, the A2H2 interval increased from 100 to $220 \mathrm{msec}$ and A2V2 from 155 to 200 msec, respectively, while the QRS complex showed gradual preexcitation (Fig. 2). The His deflection was displaced into the QRS complex and finally was buried within the QRS complex at the maximal preexcitation (Fig. 2D). The preexcited QRS morphology was identical to that of the LBBB morphology tachycardia. The AV conduction was blocked at an A1A2 interval of 200 msec.

The tachycardia was induced by the extrastimulus when $\mathrm{A} 2 \mathrm{H} 2$ was
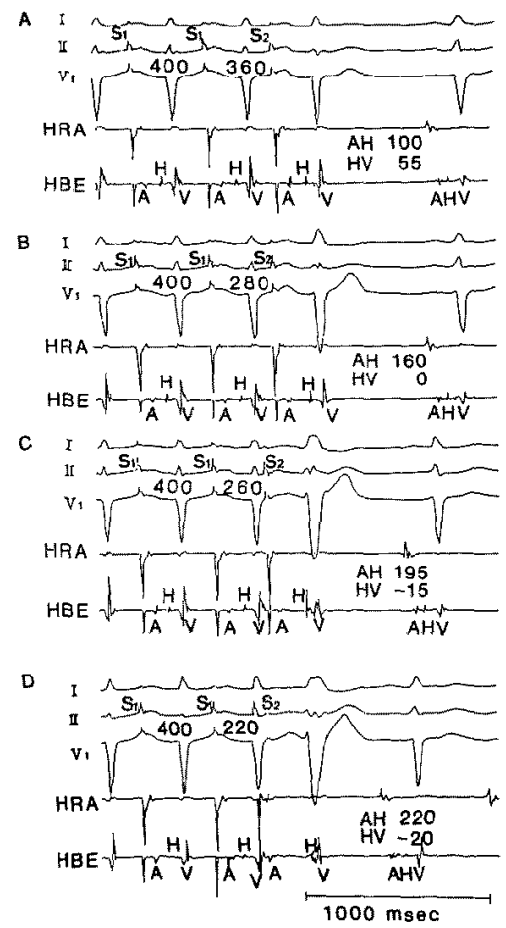

Fig. 2. Extrastimulus technique in the right atrium. A change in the QRS complex becomes evident at $360 \mathrm{msec}$ of the coupling interval of the premature stimulus (A1A2) following basic drive (A1A1 = $400 \mathrm{msec}$ ). With progressive prematurity, $\mathrm{A} 2 \mathrm{H} 2$ and $\mathrm{A} 2 \mathrm{~V} 2$ are prolonged in association with a displacement of the His deflection within the QRS complex and preexcitation of the QRS complex (A through D). At the fully preexcited QRS complex the HV interval is $-20 \mathrm{msec}$ (D). I, II and $V_{1}$ are the surface EGG leads. HRA and $\mathrm{HBE}$ are the intracavitary electrograms from the right atrium and $H$ is bundle recording site. $A, H$ and $V=$ atrial, $H$ is bundle and ventricular electrograms. 
prolonged (Fig. 2B). Premature atrial beats did not affect tachycardia (Fig. 3A). The HV interval was variable as shown in Fig. 3B. When the His deflection preceded the $\mathrm{V}$ wave, fusion was observed in the QRS complex. The VA interval changed during tachycardia but the HA interval was constant. The prolongation of the VA interval resulted in the prolongation of the cycle length of the tachycardia. During the study of the atrial stimulations, transient atrial fibrillation developed where the shortest $R R$ interval was $220 \mathrm{mscc}$ and the QRS complex was exclusively preexcited. During rapid ventricular pacing, no VA conduction was observed. The double extrastimuli were able to induce LBBB morphology tachycardia which could be terminated by the programmed stimulations. The endocardial catheter mapping of the tachycardia detected the local electrogram which preceded the QRS complex at the anterior free wall of the right ventricle. The stimulation at that site resulted in the same QRS morphology as the clinical tachycardia in the standard 12 lead surface ECG. Single premature depolarization given to the right ventricle could not reset the tachycardia.

Drug testing:

Verapamil ( $5 \mathrm{mg}$, intravenously) terminated the tachycardia with a concomitant fall in the tachycardia rate. After verapamil, tachycardia could no longer be induced. Procainamide (1,000 mg intravenously) was not effective either in terminating or preventing the induction of tachycardia. He received chronic drug therapy with verapamil $(240 \mathrm{mg} /$ day $)$ but tachycardia recurred. Atrial fibrillation also developed spontaneously and the ventricular rate was rapid, about 200 beats $/ \mathrm{min}$.

The QRS complex was exclusively preexcited and the shortest RR interval was $240 \mathrm{msec}$ in spite of therapy with verapamil. However, he had had no recurrence after an increase of the dose of verapamil to $360 \mathrm{mg} /$ day.

\section{Discussion}

Wide QRS complex tachycardia requires differential diagnosis. Six cases of AV nodal reentrant tachycardia with a bystander Mahaim fiber have been described. ${ }^{1-5)}$ The QRS complex showed LBBB and left axis deviation. Only rarely, the insertion site of the Mahaim fiber was determined intraoperatively at the anterior wall of the right ventricle. ${ }^{1)}$

In the present case, dissociation between the atrial activity and tachycardia was observed (Fig. 3A). There was no VA conduction during the rapid ventricular pacing. No paradoxical atrial capture was observed when premature ventricular depolarizations were induced by electrical stimulation during tachycardia. These findings mean that the atrium was not involved 

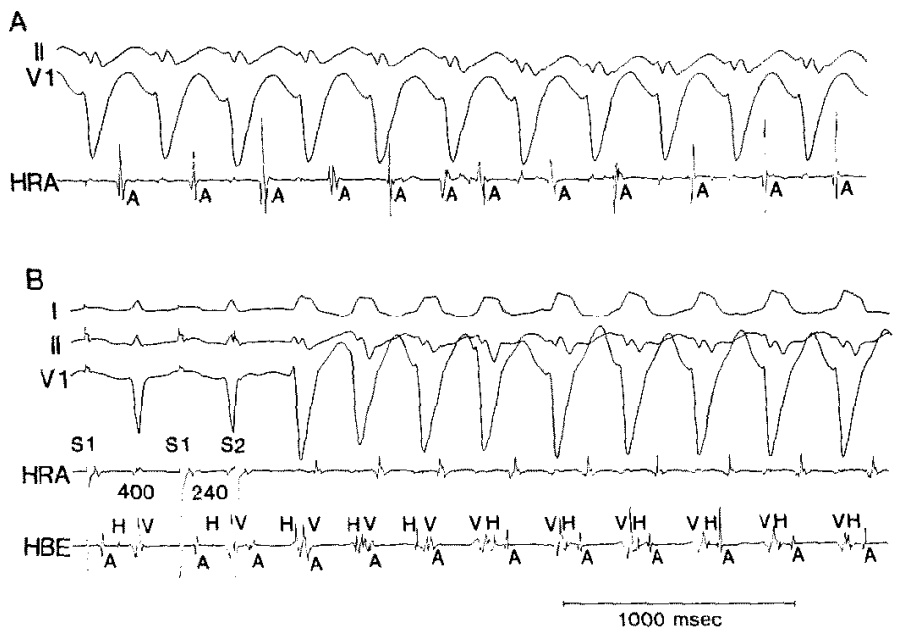

Fig. 3. Induction of wide QRS complex tachycardia. In A, ventriculoatrial dissociation is apparent when a premature atrial depolarization developed $(5,6,7$ th from the left $)$ which did not affect tachycardia. In B, tachycardia was induced by the extrastimulus method. The $\mathrm{AH}$ interval was prolonged from 70 to $195 \mathrm{msec}$, and tachycardia was induced. In the first three beats, the His deflection preceded the local ventricular depolarization and caused fusion beats as most clearly seen in lead II. From the 4th complex, the His deflection follows the $\mathrm{V}$ wave with a slightly hanging $\mathrm{VH}$ interval; then tachycardia became a little slower and cycle length became longer by $50 \mathrm{msec}$. HA interval however remained constant. Abbreviations are the same as Fig. 2.

in the reentrant circuit and the existence of an extranodal pathway was also excluded. Furthermore, it is unlikely that the His-Purkinje system' forms an active limb of the recntrant circuit becausc of the variable rclation between the His deflection and the QRS complex (Fig. 3B). Fusion was observed in the QRS complex when the His deflection preceded the QRS complex as shown in Fig. 3B. If the ventricle forms a lower common pathway, tachycardia will be easily reset by premature depolarizations of the right ventricle. However, this was not the case with our patient.

The site of origin of the tachycardia seems to be the AV node. A discontinuity obtained in the AV nodal functional curve suggests the existence of dual pathways. The $\mathrm{AH}$ interval and the $\mathrm{AV}$ interval were always prolonged over $190 \mathrm{msec}$ when tachycardia was induced by the premature atrial stimuli. Prolongation in the AV interval with progressive preexcitation means that the conduction from the atrium to the Mahaim fiber involves a slow fiber, the AV node. The site of insertion of the Mahaim fiber was determined to be the anterior wall of the right ventricle. The site seems to be similar to that determined in the intraoperative mapping study. ${ }^{11}$ The Mahaim fiber is 
classified as being of the nodo-ventricular type. Conduction delay in the limb distal to the origin of the Mahaim fiber within the AV node may explain the changing $\mathrm{HV}$ relations and a change in the cycle length of the tachycardia (Fig. 3B).

Of other clinical importance is the fact that the Mahaim fiber may have a short effective refractory period. Since the Mahaim fiber is unmasked in apparently healthy people only during supraventricular tachyarrhythmias, atrial fibrillation may lead to a similar and unexpected problem as reported in patients with Wolff-Parkinson-White syndrome. ${ }^{8)}$

\section{REFERENGES}

1. Ward DE, Camm AJ, Spurrcl RAJ: Ebstein's anomaly in association with anomalous nodoventricular conduction. Preoperative and intraoperative electrophysiologic studies. J Electrocardiol 12: 227, 1979

2. Akahtar M: Electrophysiological basis for wide QRS complex tachycardia. PACE 6: 81, 1983

3. Bardy GH, German LD, Packer MJ: Mechanism of tachycardia using nodolascicular Mahaim fiber. Am J Cardiol 54: 1140, 1984

4. Ward DE, Camm AJ, SpurreI RAJ: Ventricular preexcitation due to anomalous nodoventricular pathway. Report of 3 patients. Eur J Cardiol 9: 111, 1979

5. Morady F, Scheinman MM, Gonzales R, Hess D: His-ventricular dissociation in patients with reciprocating tachycardia and a nodoventricular bypass tract. Circulation 64: 839, 1982

6. Aizawa $Y$, Oda $H$, Satoh M, Shibata A: Transient entrainment of ventricular tachycardia with continuous electrical activity. Am Heart J 114: 154, 1987

7. Aizawa Y, Satoh M, Suzuki K, Aizawa M, Funazaki T, Shibata A, Josephson ME: Early experiences of endocardial catheter mapping of the left ventricle in patients with sustained ventricular tachycardia. Jpn Circ J 51: 1283, 1987

8. Dreifus LS, Haiat R, Watanabe Y: Ventricular fibrillation. A possible mechanism of sudden death in Wolf-Parkinson-White syndrome. Circulation 43:520, 1971 